\title{
Epitaxial lateral overgrowth of InP on Si from nano-openings: Theoretical and experimental indication for defect filtering throughout the grown layer
}

\author{
F. Olsson, ${ }^{1, a)}$ M. Xie, ${ }^{1}$ S. Lourdudoss, ${ }^{1}$ I. Prieto, ${ }^{2}$ and P. A. Postigo ${ }^{2}$ \\ ${ }^{1}$ School of Information and Communication Technology, Royal Institute of Technology, Electrum 229, S-164 \\ 40 Kista, Sweden \\ ${ }^{2}$ Instituto de Microelectrónica de Madrid, Centro Nacional de Microelectrónica (IMM-CNM-CSIC), Isaac \\ Newton 8, E-28760, Tres Cantos, Madrid, Spain
}

(Received 14 January 2008; accepted 14 July 2008; published online 12 November 2008)

\begin{abstract}
We present a model for the filtration of dislocations inside the seed window in epitaxial lateral overgrowth (ELO). We found that, when the additive effects of image and gliding forces exceed the defect line tension force, filtering can occur even in the openings. The model is applied to ELO of $\mathrm{InP}$ on $\mathrm{Si}$ where the opening size and the thermal stress arising due to the mask and the grown material are taken into account and analyzed. Further, we have also designed the mask patterns in net structures, where the tilting angles of the openings in the nets are chosen in order to take advantage of the filtering in the openings more effectively, and to minimize new defects due to coalescence in the ELO. Photoluminescence intensities of ELO InP on Si and on InP are compared and found to be in qualitative agreement with the model. () 2008 American Institute of Physics. [DOI: $10.1063 / 1.2977754]$
\end{abstract}

\section{INTRODUCTION}

Heteroepitaxy of GaAs or InP on Si has been a topic of intense research for a long time and has received renewed interest recently for silicon photonics. The lattice mismatch between $\mathrm{GaAs}$ and $\mathrm{Si}$ is $\sim 4 \%$ and that between $\mathrm{InP}$ and $\mathrm{Si}$ is $\sim 8 \%$. Such a high mismatch results in the generation of misfit dislocations. An important method for filtering these dislocations has been the use of an epitaxial lateral overgrowth (ELO), where the growth proceeds laterally from the windows of the seed material cut through a mask layer, which is often $\mathrm{SiO}_{2}$ or $\mathrm{SiN}_{x}$. The mask layer will block the propagation of the underlying dislocations, creating an almost dislocation-free layer, except above and in the vicinity of the seed windows. The ELO method has been widely used for heteroepitaxial growth of $\mathrm{GaAs}$ on $\mathrm{Si}^{1}$ and $\mathrm{GaN}$ on sapphire. ${ }^{2}$ It has also been employed for InP on Si by Naritsuka and Nishinaga ${ }^{3}$ and by our group ${ }^{4,5}$ in the recent years with relative success. However, in order to achieve a nearly dislocation-free continuous and homogeneous layer overall above the mask and the opening, there are three main problems to overcome: (a) the threading dislocation may still propagate up to the overlying layer through the seed windows, (b) generation of new defects such as dislocations, twins, and voids in the region of coalescence where different growth fronts meet and start to merge, and (c) thermal cracking due to differences in thermal expansion coefficients between the mask and the layer; this may occur for thick layers combined with large tensile strain with a predicted Griffith crack thickness of about $4 \mu \mathrm{m}$ at $0.2 \%$ strain. $^{6}$ One way to reduce the number of propagating dislocations through the seed window is to make the seed windows sufficiently small in the nanometer range. Under such a condition, the openings

${ }^{a)}$ Electronic mail: folsson1@ @ kth.se. should also result in the filtering of defects owing to the additive effects of image force ${ }^{7}$ and strain-induced gliding force ${ }^{8}$ that would dominate the line tension of the misfit segments of the dislocation. Smaller seed windows also open up for less spacing between the openings and thereby a possibility of reducing the layer thickness so that no thermal cracking will occur. A way to control and reduce the problems with the coalescence process has been to align the seed windows in proper directions which will lead to a v-shaped merging process, the so-called zipper effect, where the initial growth takes place in a corner and follows two inclined opening lines, which, by lateral growth, closes the masked area in between with a minimal introduction of new dislocations. ${ }^{9}$ In this article, we present a model for the filtration of dislocations within the small seed window openings by exemplifying $\mathrm{ELO}$ of $\mathrm{InP}$ on $\mathrm{Si}$, the results of which are in qualitative agreement with the model. In the experiments, we have also taken into account the zipper effect in the mask design for nano-ELO. The laterally grown InP layer is only $1.5 \mu \mathrm{m}$ thick and exhibits room temperature photoluminescence (PL) intensity between $5 \%$ and $10 \%$ of that of InP grown on planar InP substrate.

\section{THEORY}

According to Rehder et al. ${ }^{10}$ a mask surface such as that of silicon dioxide can act as a free surface and, as such, attracts the dislocations lying in the immediate proximity of the interface through the mirror image force. ${ }^{7}$ The driving force in the semiconductor material for such a dislocation motion is the attractive strain force on threading segments, so as to withhold them near the mask surface. For the semiconductor material, this image force $F_{I}$ may be written as ${ }^{11}$ 


$$
F_{I}=\frac{G b^{2} h}{4 \pi r \cos \phi}\left(\cos \varphi+\frac{\sin \varphi}{(1-\nu)}\right)
$$

where $G$ is the shear modulus, $b$ is the length of the Burgers vector for the threading dislocation, $h$ is the thickness of the mask, $v$ is Poisson's ratio, $\phi$ is the angle between the threading segments and the interface, $r$ is the distance along a (111) glide plane from a sidewall, and $\varphi$ is the angle between the dislocation line vector and the Burgers vector. $F_{L}$, the line tension of the misfit segment of the dislocation, is given by ${ }^{8}$

$$
F_{L}=\frac{G b^{2}\left(1-\nu \cos ^{2} \varphi\right)}{4 \pi(1-\nu)}[\ln (h / b+1)]
$$

Both $F_{I}$ and $F_{L}$ will be acting on the part of growth taking place initially in the seed windows and starting to grow laterally. In the layer resulting out of the ELO, there will also be a thermal strain due to its difference in thermal expansion coefficient with respect to that of the mask layer. ${ }^{12}$ Thermal strain from the substrate should also be taken into account. However, the thermal strain from the substrate can be neglected at temperatures close to the seed layer growth temperature, as in the case of our experimental conditions. The thermal strain between the layer and the mask may cause a displacement or gliding (however small) of the semiconductor material with respect to the mask layer at the interface. However, it is limited in our case; the resulting thermal stress field around the mask will be significant with a large part taken up by the semiconductor material even for the cases where the laterally grown layer is much thicker than the mask layer. ${ }^{13}$ This points to an existing stress field around the mask before the ELO starts. For small seed windows, this stress field will render the initially grown layer in the opening strained in the immediate proximity of the mask both inside and outside the opening. We assume that the seed windows are small enough so that the induced strain in the initial growth within the seed windows is constant.

For ELO, the masking layer often consists of line openings aligned in favorable directions that would enable a high lateral growth. In the case where $a_{\text {layer }}$, the thermal expansion coefficient of the semiconductor layer, is superior to $a_{\text {mask }}$, the thermal expansion coefficient of the mask, the thermal strain experienced by the initial growth layer in the seed window will be compressive at the growth temperature, mainly in the direction perpendicular to the mask walls. The induced strain on the grown material in the seed window will then result in a gliding force $F_{G}$ that will act on the threading dislocations according to ${ }^{8}$

$$
F_{G}=\frac{2 G b h(1+\nu) \cos \lambda}{1-\nu} \varepsilon,
$$

where $\lambda$ is the angle between the Burgers vector and the line in the interface plane that is perpendicular to the intersection of the glide plane with the interface. $\varepsilon$ is the thermal strain, which is given by
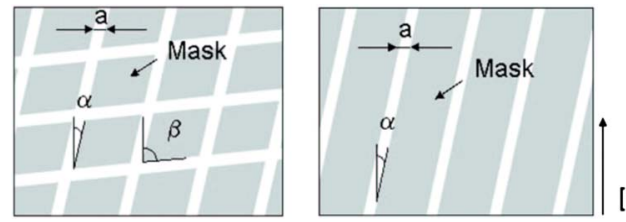

[110]

FIG. 1. (Color online) Left: net with openings, $a=200$ or $1000 \mathrm{~nm}$ and the tilts of the lines with angles $\alpha$ and $\beta=15^{\circ}$ and $60^{\circ}, 15^{\circ}$ and $75^{\circ}, 15^{\circ}$ and $105^{\circ}, 15^{\circ}$ and $120^{\circ}, 30^{\circ}$ and $60^{\circ}, 30^{\circ}$ and $75^{\circ}, 30^{\circ}$ and $105^{\circ}$, and $30^{\circ}$ and $120^{\circ}$, respectively. Right: lines with openings, $a=200$ or $1000 \mathrm{~nm}$ and with $\alpha=15^{\circ}, 30^{\circ}, 60^{\circ}, 75^{\circ}, 105^{\circ}$, and $120^{\circ}$. The separation between openings is $5 \mu \mathrm{m}$.

$$
\varepsilon=\int\left(\alpha_{\text {layer }}-\alpha_{\text {mask }}\right) d T .
$$

Here, the integration is done from the growth temperature $\left(T_{G}\right)$ to the deposition temperature of the mask $\left(T_{\text {mask }}\right)$. If the thermal expansion coefficients are considered to be independent of temperature, then, Eq. (4) is simplified as

$$
\varepsilon \approx\left(\alpha_{\text {layer }}-\alpha_{\text {mask }}\right)\left(T_{G}-T_{\text {mask }}\right) \text {. }
$$

The experienced total force on the dislocations in the grown film in the seed window will then consist of three terms, $F_{I}$, $F_{L}$, and $F_{G}$. When $F_{I}+F_{G}>F_{L}$ is fulfilled, the dislocations above the openings will start to glide toward the sidewalls. At which distance $r$ this would happen can be directly derived by combining Eqs. (1)-(3) and can be written as

$$
r=\frac{\frac{h}{\cos \phi}[(1-\nu) \cos \varphi+\sin \varphi]}{\left(1-\nu \cos ^{2} \varphi\right)[\ln (h / b+1)]-\frac{8 \pi h}{b}(1+\nu) \cos \lambda \varepsilon} .
$$

This will be used below to analyze the experimental results.

\section{EXPERIMENTAL}

In order to demonstrate the model outlined above, we have designed a set of patterns consisting of seed windows of different widths, including sufficiently small widths, to take advantage of the additive effects of $F_{I}$ and $F_{G}$ over $F_{L}$.

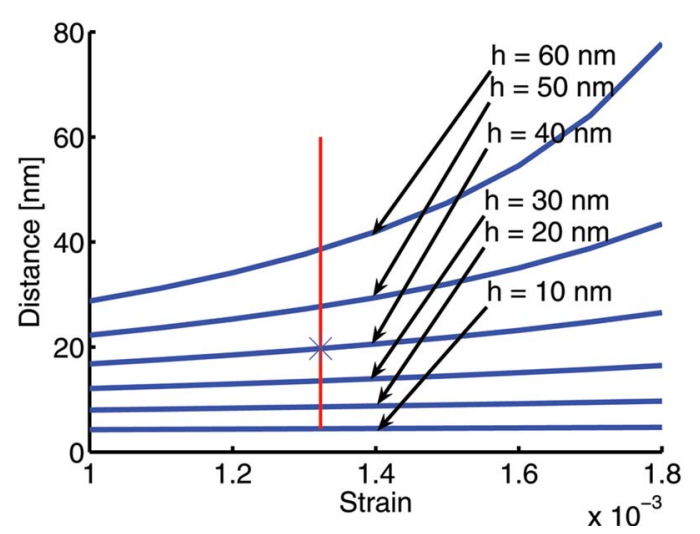

FIG. 2. (Color online) Calculated curves from Eq. (6): distance $r$ from the side wall of the mask opening where filtering of dislocations takes place as a function of thermal strain between the grown layer and the mask for different mask thicknesses $h$ (which is also the grown layer thickness in the opening). The vertical line corresponds to the existing strain in our experiment and the cross for the specific case of the mask thickness $40 \mathrm{~nm}$. 


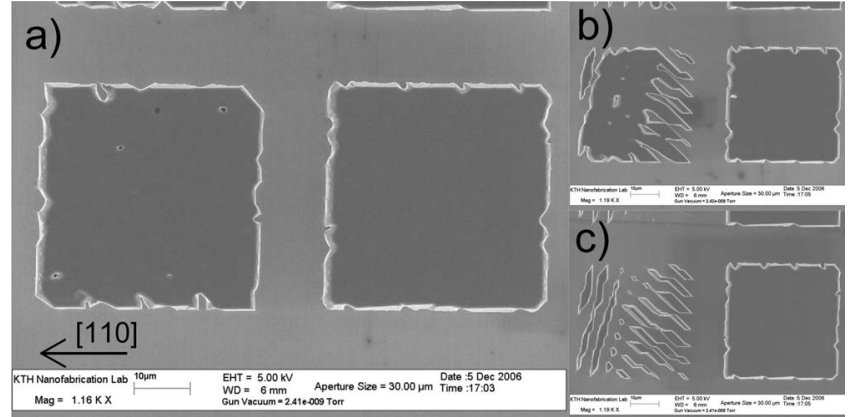

FIG. 3. SEM pictures of sample (a) with net patterns of tilting angles $15^{\circ}$ and $120^{\circ}$ : (a) patterns with openings of 200 (left) and $1000 \mathrm{~nm}$ (right) after matured growth, [(b) and (c)] patterns with openings of 200 (left) and 1000 $\mathrm{nm}$ (right) after less matured growth

Further, we have also divided the patterns into two groups. The first group consists of a net with two sets of line openings with different tilting angles and, for comparison, the second group with parallel line openings only. The net design is to enable the zipper effect mentioned above. A schematic view of the patterns can be seen in Fig. 1. Each group of patterns was contained in a field of size $40 \times 40 \mu \mathrm{m}^{2}$. Both of these patterns were formed on samples A and B. Sample A was $\mathrm{Si}$ wafer (off-cut $4^{\circ}$ toward [111]) precoated with seed $\mathrm{InP}$ (containing a very high concentration of dislocations) by metal organic vapor phase epitaxy and sample B was plain InP substrate with a misorientation of $2^{\circ}$ toward [110]. The patterns were prepared by combining plasma enhanced chemical vapor deposition of $\mathrm{SiO}_{2}$, electron beam lithography, and reactive ion beam etching (RIBE) techniques. The $\mathrm{SiO}_{2}$ layer thickness was $40 \mathrm{~nm}$ and deposited at $300{ }^{\circ} \mathrm{C}$. The constituting line openings of the net are either 200 or $1000 \mathrm{~nm}$ wide. The separation of the openings is $5 \mu \mathrm{m}$ in both directions of the net. The tilt angles of the lines in the net, $\alpha$ and $\beta$, are $15^{\circ}$ and $60^{\circ}, 15^{\circ}$ and $75^{\circ}, 15^{\circ}$ and $105^{\circ}$, $15^{\circ}$ and $120^{\circ}, 30^{\circ}$ and $60^{\circ}, 30^{\circ}$ and $75^{\circ}, 30^{\circ}$ and $105^{\circ}$, and $30^{\circ}$ and $120^{\circ}$, respectively. All these angles are defined with respect to the [110] direction of the wafer. The second group of line patterns has openings of the same width as above along $\alpha=15^{\circ}, 30^{\circ}, 60^{\circ}, 75^{\circ}, 105^{\circ}$, and $120^{\circ}$. After patterning, a postsequential ELO of InP on samples A and B was conducted in an Aixtron low pressure hydride vapor phase epitaxy reactor in nitrogen ambient. The total pressure was 20 mbar and the partial pressures of $\mathrm{InCl}$ and $\mathrm{PH}_{3}$ were 2.8 and 0.28 mbar, respectively. The growth temperature was, in

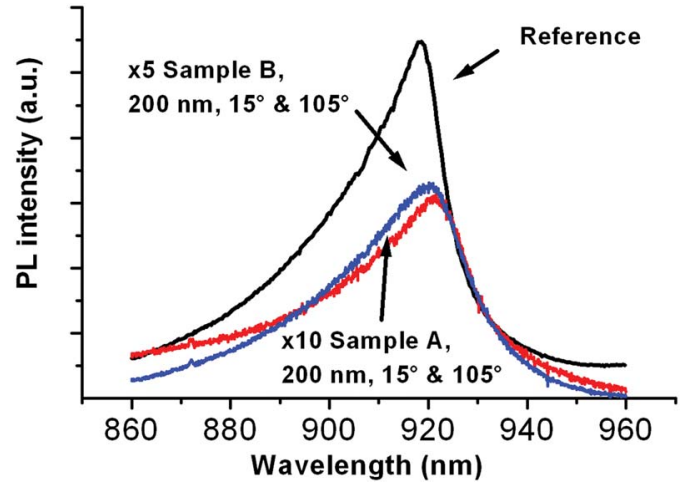

FIG. 4. (Color online) Micro-PL spectrum of ELO InP grown on net structures on sample $\mathrm{A}(\mathrm{InP} / \mathrm{Si})$ and sample $\mathrm{B}(\mathrm{InP} / \mathrm{InP})$, and of the reference planar sample (InP/InP). Samples A and B had the opening size of $200 \mathrm{~nm}$ and tilting angles of $15^{\circ}$ and $105^{\circ}$.

both cases, $615{ }^{\circ} \mathrm{C}$ and the growth time was $2 \min 15 \mathrm{~s}$ which resulted in a $1.5 \mu \mathrm{m}$ thick InP layer on the patterns. The lateral growth rates is crystal orientation dependent and ranged in a subsequent experiment, a $1.5 \mu \mathrm{m}$ thick $\mathrm{InP}$ was grown on a plain InP substrate using the above growth parameters, yielding a growth rate of $10 \mu \mathrm{m} / \mathrm{h}$ and was used as a reference sample. All the grown samples were subjected to micro-PL measurements at room temperature using a $\mathrm{HeNe}$ laser $(\lambda=632.8 \mathrm{~nm})$ as the excitation source. The probe size diameter is $2 \mu \mathrm{m}$ and the stage can be moved in steps down to $0.5 \mu \mathrm{m}$. The samples were mapped in rectangular regions with step sizes of $1-1.2 \mu \mathrm{m}$ in regions close to the center of the patterns or in regions of complete growth coverage. The total number of data points ranged from about $100-150$ for patterns with $200 \mathrm{~nm}$ seed windows up to about 400 for patterns with $1000 \mathrm{~nm}$ seed windows. Smaller regions with a complete growth coverage on the patterns with $200 \mathrm{~nm}$ openings caused the mapping areas to be reduced in size. The problems with incomplete growth regions were particularly important for the line patterns. The peak intensity average from these mapping points is presented as well as the maximum peak intensity. The opening sizes used here were fed into our model described above, analyzed, and compared to the PL data.

\section{RESULTS AND DISCUSSION}

In Fig. 2, by using Eq. (6), the distance $r$ from the sidewall inside the opening where the line tension force is over-

TABLE I. PL data for maximum PL intensity and average maximum PL intensity of the mapped region with respect to that of the reference sample. FWHM values and wavelength values correspond to the PL intensity maximum. Data for ELO InP grown on both net and line structures of samples A and B with an opening size of $200 \mathrm{~nm}$ is presented.

\begin{tabular}{lccccccc}
\hline \hline & \multirow{2}{*}{$\begin{array}{c}\text { Net (opening: } 200 \mathrm{~nm}) \\
\text { Sample A }\end{array}$} & \multicolumn{2}{c}{ Lines (opening: 200 nm) } & Ref. \\
& \multicolumn{2}{c}{ Sample A } & Sample B & Planar \\
\hline Angle(s) & $15^{\circ}-105^{\circ}$ & $15^{\circ}-120^{\circ}$ & $15^{\circ}$ & $105^{\circ}$ & $15^{\circ}$ & $105^{\circ}$ & layer \\
Max. int. (\%) & 6.4 & 5.7 & 2 & 5 & 6.1 & 5 & \\
Av. int. (\%) & 5.2 & 4.9 & 1.7 & 4 & 5.8 & 4.3 & 100 \\
$\lambda(\mathrm{nm})$ & 922 & 921 & 923 & 922 & 920 & 921 & 918 \\
FWHM (nm) & 28 & 29 & 31 & 33 & 25 & 26 & 26 \\
\hline \hline
\end{tabular}


TABLE II. PL data with maximum PL intensity and average maximum PL intensity of the mapped region with respect to that of the reference sample. FWHM values and wavelength values correspond to the PL intensity maximum. PL data for the ELO InP grown on net structures of sample B with an opening size of $200 \mathrm{~nm}$ is presented.

\begin{tabular}{lcccccc}
\hline \hline \multicolumn{7}{c}{ Net (opening 200 nm) } \\
Sample B \\
\hline Angles & $15^{\circ}-105^{\circ}$ & $15^{\circ}-120^{\circ}$ & $30^{\circ}-60^{\circ}$ & $30^{\circ}-75^{\circ}$ & $30^{\circ}-105^{\circ}$ & $30^{\circ}-120^{\circ}$ \\
Max. int. (\%) & 12 & 9.2 & 5.6 & 7.9 & 5.4 & 5.0 \\
Av. int. (\%) & 9.2 & 6.2 & 4.7 & 5.9 & 4.3 & 4.3 \\
$\lambda(\mathrm{nm})$ & 921 & 919 & 920 & 920 & 921 & 920 \\
FWHM (nm) & 30 & 33 & 27 & 29 & 31 & 29 \\
\hline \hline
\end{tabular}

come by the sum of image and the gliding forces is shown as a function of thermal strain for different mask layer thicknesses $h$. In plotting these curves, we have used the relevant values for the zinc blende structure, namely, $b=4 \AA, \varphi$ $=60^{\circ}, \lambda=60^{\circ}$, and $\phi=35.3^{\circ}$ in Eq. (6). As $h$ increases, i.e., as the thickness of the grown layer within the seed window increases toward the critical thickness, the two terms in the denominator become equal and Eq. (6) yields infinite values of $r$.

In the same figure, we have incorporated also our experimental parameters. The vertical line corresponds to the existing thermal strain between the $\mathrm{SiO}_{2}$ mask and the grown InP layer in our experiment, and the cross for the specific case of the mask thickness $40 \mathrm{~nm}$ used in our experiments. The thermal strain of $0.13 \%$ is derived from Eq. (5) knowing $\alpha_{\text {InP }}$ and $\alpha_{\text {mask }}$ as 4.75 and $0.55 \times 10^{-6} \mathrm{~K}^{-1}$, respectively, and $\Delta T=315 \mathrm{~K}$. Thus, effective filtering takes place at $r$ $\sim 20 \mathrm{~nm}$ (corresponding to the cross in Fig. 2). This demands an opening size of $\sim 40 \mathrm{~nm}$. A significant dislocation filtering may be expected for even smaller opening sizes, whereas, the effect on larger openings is more of a pure edge effect. It is possible to elucidate the filtering effect by means of micro-PL measurements.

Figure 3 presents a scanning electron microscope (SEM) view of ELO InP on $\mathrm{Si}$ on the net patterns of tilting angles $15^{\circ}$ and $120^{\circ}$ after matured growth (a) and less matured growth [(b) and (c)]. In (a)-(c), the left pattern has $200 \mathrm{~nm}$ openings and the right has $1000 \mathrm{~nm}$ openings. The less matured growth is from the same sample but at a position which is affected by the drainage of growth species at the upper side. Figures 3(b) and 3(c) exemplify the growth evolving process in the net structures. The growth starts in the crossing points of the line openings. These crossing points are slightly enlarged due to the double exposure by the e-beam. The growth continues according to a zipper growth mode and the extending lateral growth leads to a closure of the mask area in between the openings.

The measured micro-PL spectra of net structures with the openings of $200 \mathrm{~nm}$ at $\alpha$ and $\beta$ of $15^{\circ}$ and $105^{\circ}$, respectively, for both samples A and B are shown in Fig. 4. In the same plot, the spectrum for the reference planar sample InP/ $\mathrm{InP}$ (subs) is also given. All the grown layers were $1.5 \mu \mathrm{m}$ thick and unintentionally doped. The PL intensities of samples A and B are of the same magnitude indicating that the growth behavior on the patterned surface is comparable in the case of both InP/Si and InP/InP; with respect to the reference sample, their intensities are 5\% and 10\%, respectively. A reduction in intensity for sample $\mathrm{B}$, with respect to the reference sample, is likely to be mainly due to the surface morphology of the growth conducted on the patterned surface, which, however, can be improved by growth optimization or subsequent chemical and mechanical polishing. ${ }^{14} \mathrm{~A}$ second source of intensity lowering might come from dislocations generated by thermal stress originating from the difference in thermal expansion coefficients between the layer and the mask. Nevertheless, the full width at half maximum (FWHM) for both A and B are comparable with that of planar InP, which is an indication of high quality ELO InP layers grown on $\mathrm{Si}$ and InP. Referring to our model, the cross in Fig. 2 demands an opening size of $2 \times 20 \mathrm{~nm}^{2}$, i.e., $40 \mathrm{~nm}$. Our opening size is $200 \mathrm{~nm}$, and only $20 \mathrm{~nm}$ from each side of the sidewalls is expected to be affected by the image forces in combination with the thermal strain force. This means that we anticipate only about $20 \%$ filtering in the whole opening if the defects originate from the openings.

In Tables I-IV, the PL data of other structures with the opening sizes of 200 and $1000 \mathrm{~nm}$ are summarized, respectively. Tables I and II contain PL data for both samples A and $\mathrm{B}$, respectively, in which the opening size is $200 \mathrm{~nm}$. Included in Table I is also some data from line patterns. Tables III and IV contain data for $1000 \mathrm{~nm}$ opening for samples A and $\mathrm{B}$, respectively. The patterns of smaller opening were not fully resolved in our lithographic step. This was particularly the case for the smaller openings of sample A, probably caused by less thickness homogeneity of the resist due to the larger surface roughness of the InP seed. Besides, some areas were more affected or completely missing, which was the case for many line patterns and nets on sample A with angles

TABLE III. PL data with maximum PL intensity and average maximum PL intensity of the mapped region with respect to that of the reference sample. FWHM values and wavelength values correspond to the PL intensity maximum. PL data for ELO InP grown on $1000 \mathrm{~nm}$ wide openings for sample A is presented.

\begin{tabular}{lcccc}
\hline \hline \multicolumn{5}{c}{ Net (opening: $1000 \mathrm{~nm})$} \\
Sample A \\
\hline Angles & $15^{\circ}-105^{\circ}$ & $15^{\circ}-120^{\circ}$ & $15^{\circ}-60^{\circ}$ & $15^{\circ}-75^{\circ}$ \\
Max. int. (\%) & 4.8 & 4.2 & 2.8 & 2.9 \\
Av. int. (\%) & 3.4 & 3.2 & 2.3 & 2.2 \\
$\lambda(\mathrm{nm})$ & 922 & 923 & 921 & 921 \\
FWHM (nm) & 34 & 27 & 33 & 28 \\
\hline \hline
\end{tabular}


TABLE IV. PL data with maximum PL intensity and average maximum PL intensity of the mapped region with respect to that of the reference sample. FWHM values and wavelength values correspond to the PL intensity maximum. PL data for ELO InP grown on $1000 \mathrm{~nm}$ wide openings for sample B is presented.

\begin{tabular}{lcccccccc}
\hline \hline \multicolumn{7}{c}{ Net (opening: $1000 \mathrm{~nm})$} \\
Sample B \\
\hline Angles & $15^{\circ}-60^{\circ}$ & $15^{\circ}-75^{\circ}$ & $15^{\circ}-105^{\circ}$ & $15^{\circ}-120^{\circ}$ & $30^{\circ}-60^{\circ}$ & $30^{\circ}-75^{\circ}$ & $30^{\circ}-105^{\circ}$ & $30^{\circ}-120^{\circ}$ \\
Max. int. (\%) & 6.2 & 9.2 & 5.5 & 11.8 & 8.0 & 7.4 & 6.2 & 4.6 \\
Av. int. (\%) & 4.5 & 4.5 & 4.7 & 5.5 & 4.3 & 3.8 & 3.7 & 4.0 \\
$\lambda$ (nm) & 919 & 919 & 920 & 920 & 920 & 920 & 918 & 920 \\
FWHM (nm) & 29 & 32 & 30 & 33 & 29 & 27 & 31 & 25 \\
\hline \hline
\end{tabular}

$30^{\circ}-X^{\circ}$. By comparing Tables I-IV, one notes that the highest intensities are achieved for the net structures consisting of lines in the direction of $15^{\circ}$ and $105^{\circ}$, and $15^{\circ}$ and $120^{\circ}$. The (average) intensity for sample B (patterned $\mathrm{InP} / \mathrm{InP}$ ) is to a large extent constant for both opening sizes (200 and 1000 $\mathrm{nm}$ ) but with an enhancement of about $10 \%$ for the smaller openings. The surface damage arising out of the openings is less proportional to the size of the openings and hence less for the small openings. As a result, small openings exhibit higher intensity. However, the net with tilting angles $15^{\circ}$ and $105^{\circ}$ shows a remarkable high intensity, the cause of which has not been identified. The tendency is an increased average intensity of about $50 \%$ for the smaller opening size for sample A (the patterned InP/Si). This is in qualitative agreement with the outlined model above which allows for larger filtering for smaller openings in sample $\mathrm{A}$, which in turn would lead to a higher PL intensity because of the less number of dislocations. Relatively, a constant PL intensity irrespective of the opening size (200 or $1000 \mathrm{~nm}$ ) is only expected for sample B as there exist no misfit dislocations to filter. Besides, enhanced intensity for the nets with $\alpha$ and $\beta$ of $15^{\circ}$ and $105^{\circ}$, and $15^{\circ}$ and $120^{\circ}$ with respect to the other sets of investigated $\alpha$ and $\beta$ is indicative of a more efficient filtering of dislocations lying in slipping planes whose projection in the (001) surface plane is perpendicular to the [110] direction, which enlarges the distance $r$ to the sidewall for openings lying farther off from the [110] direction, again in conformity with the model. The second reason may rely on the asymmetry between dislocations between (111)A planes and (111)B planes. ${ }^{4}$ From Ref. 4 it is understood that the growth rates are caused by higher threading dislocation density at the (111)B plane in the InP seed. When selective area growth (SAG) was initiated in the openings aligned at [1-10] direction, the growth is bounded by (111)A planes. The threading dislocation lines in the InP seed lying on the (111)B plane will exit from this boundary plane and intersect the (111)A plane. This causes nucleation sites for further growth on the (111)A plane. According to the BurtonCabrera-Frank (BCF) mechanism, the growth is liable to occur in the form of spiral growth and, hence, the (111)A plane will be a high growth rate plane. The merging of a less dislocation containing growth front (111)A arriving from openings close to [1-10] with the highly dislocation filtered material from openings close to [110] would be beneficial to the overall reduction in dislocation density. In Table I, some line structures are also collected. The tendency is a lower PL intensity for them compared to similar net structures. This is in agreement with the supposed different merging process, which would resemble the zipper effect ${ }^{9}$ for the net structures. In all the samples, the wavelength is slightly redshifted compared to the reference sample. This is most probably due to a certain thermal strain from the silicon substrate which remains from the original seed layer growth since the dislocation glide velocities is thermally activated and the strain cannot usually be fully relaxed. The smaller expansion coefficient of silicon can lead to a tensile strained InP seed.

\section{CONCLUSION}

In summary, we have analyzed the defect filtering mechanism inside the opening in ELO of InP on silicon. We found that, when the additive effects of image and gliding forces exceed the defect line tension force, filtering can occur even in the openings. However, in order to realize this, the opening size and the thermal stress arising due to the mask and the grown material are to be taken into account; we have analyzed and presented this correlation. PL intensities of ELO InP on Si and on InP are compared and found to be in qualitative agreement with the model. The net pattern structures take advantage of the zipper effect to minimize defects due to coalescence; the tilting angles of the openings in the net structures are also chosen in order to take advantage of the filtering in the openings more effectively. Openings smaller than those considered here should enable an even better quality of ELO InP above the openings.

\section{ACKNOWLEDGMENTS}

We would like to acknowledge A. Grishin for fruitful discussion and F. Gerard and A. R. Alija for help with the e-beam lithography and RIBE fabrication. The project was partly funded by the Swedish Foundation for Strategic Research and EU NoEs PHOREMOST (Grant No. IST-2511616-NOE), SANDIE (Grant No. NMP4-CT-2004500101), and by the Spanish MEC and CAM through projects NANOSELFII (Grant No. TEC-2005-05781-C0301), MEC (Grant No. MAT2005-01388), Grant Nos. NAN2004-09109-C04, NAN2004-08843-C05-04, Consolider-CSD 2006-19, and CAM S-0505-/ESP-0200.

${ }^{1}$ Y. Ujiie and T. Nishinaga, Jpn. J. Appl. Phys., Part 2 28, L337 (1989).

${ }^{2}$ T. S. Zheleva, O.-H. Nam, M. D. Bremser, and R. F. Davis, Appl. Phys. Lett. 71, 2472 (1997).

${ }^{3}$ S. Naritsuka and T. Nishinaga, J. Cryst. Growth 146, 314 (1995).

${ }^{4}$ Y. T. Sun, J. Napierala, and S. Lourdudoss, Proceedings of the 14 th International Conference on Indium Phosphide and Related Materials (IEEE, 
Stockholm, Sweden, 2002), p. 339.

${ }^{5}$ Y. T. Sun and S. Lourdudoss, Proceedings of the Photonics Packaging and Integration III (SPIE, Bellingham, WA, 2003), Vol. 4997, p. 221.

${ }^{6} \mathrm{~J}$. E. Ayers, Heteroepitaxy of Semiconductors Theory, Growth and Characterization (CRC, Boca Raton, FL/Taylor \& Francis, London, 2007), p. 241.

${ }^{7}$ D. Hull and D. J. Bacon, Introduction to Dislocations, 4th ed. (Butterworth-Heinemann, Oxford, 2001).

${ }^{8}$ J. W. Matthews, S. Mader, and T. B. Light, J. Appl. Phys. 41, 3800 (1970).

${ }^{9}$ Z. Yan, Y. Hamaoka, S. Naritsuka, and T. Nishinaga, J. Cryst. Growth 212 ,
1 (2000).

${ }^{10}$ E. M. Rehder, C. K. Inoki, T. S. Kuan, and T. F. Kuech, J. Appl. Phys. 94, 7892 (2003)

${ }^{11}$ X. G. Zhang, P. Li, G. Zhao, D. W. Parent, F. C. Jain, and J. E. Ayers, J. Electron. Mater. 27, 1248 (1998).

${ }^{12}$ M. Yamaguchi, M. Tachikawa, M. Sugo, S. Kondo, and Y. Itoh, Appl. Phys. Lett. 56, 27 (1990).

${ }^{13}$ Y. T. Sun, K. Baskar, and S. Lourdudoss, J. Appl. Phys. 94, 2746 (2003).

${ }^{14}$ S. Saravanan, Y. Hayashi, T. Soga, T. Jimbo, M. Umeno, N. Sato, and T. Yonehara, J. Cryst. Growth 237-239, 1450 (2002). 